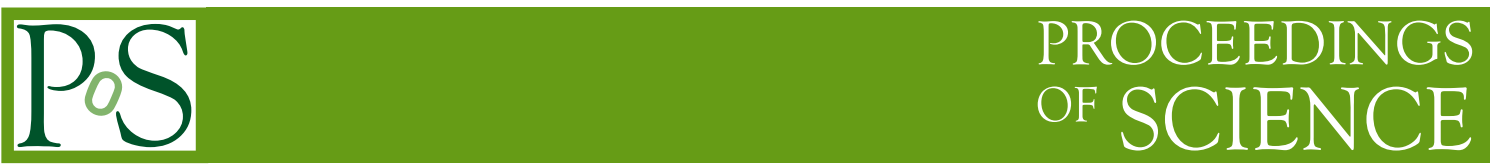

\title{
Science with the Southern Gamma-ray Survey Observatory
}

\author{
Fabian Schüssler* \\ IRFU, CEA, Université Paris-Saclay, F-91191 Gif-sur-Yvette, France \\ E-mail: fabian.schusslerecea.fr
}

\section{on behalf of the SGSO Alliance}

\begin{abstract}
The Southern Gamma-ray Survey Observatory (SGSO) is a proposed next-generation extensive air shower detector located at a high-altitude site in the mountains of South America. SGSO will be sensitive to astrophysical gamma rays and cosmic rays in the energy range between $100 \mathrm{GeV}$ and $100 \mathrm{TeV}$. Its science case is build on three main pillars: SGSO will allow to monitor the high-energy transient sky, unveil Galactic particle acceleration at the highest energies and probe physics beyond the standard model.

A collection of scientists interested in the science and engineering of SGSO formed the Southern Gamma-ray Survey Observatory Alliance (sgso-alliance.org) with the aim to elucidate the expected scientific discoveries of SGSO and the design parameters required to obtain these discoveries. The Alliance currently explores several detector design options via simulations and prototyping of various elements of the detectors and assesses the suitability of possible sites for the detector deployment.

This contribution will provide an overview over the science case and potential reach of SGSO. We will especially highlight expected contributions of SGSO to the era of multi-messenger timedomain astronomy at the highest energies like searches for counterparts to high-energy neutrinos and gravitational waves. We will also discuss the important synergies and complementarities with current and planned observatories like the Cherenkov Telescope Array and LHAASO.
\end{abstract}

36th International Cosmic Ray Conference -ICRC2019-

July 24th - August 1st, 2019

Madison, WI, U.S.A.

${ }^{*}$ Speaker. 
The content of this contribution is based on the description of the science case for a wide field-of-view very-high-energy gamma-ray observatory in the Southern hemisphere [1].

\section{References}

[1] A. Albert, R. Alfaro, H. Ashkar, C. Alvarez, J. Álvarez, J. C. Arteaga-Velázquez et al., Science Case for a Wide Field-of-View Very-High-Energy Gamma-Ray Observatory in the Southern Hemisphere, arXiv e-prints (2019) arXiv:1902.08429 [1902.08429]. 